\title{
Note on Transliteration: \\ Franco-Khmer Transcription System of Franklin E. Huffman
}

Transcriptions in this book follow the Franco-Khmer transcription system developed by Franklin E. Huffman in 1983 .

\begin{tabular}{|c|c|c|c|c|c|}
\hline \multirow{2}{*}{$\begin{array}{l}\text { Consonants } \\
\text { Ist }\end{array}$} & \multicolumn{2}{|c|}{ Transcription } & \multirow[t]{2}{*}{ Vowels } & \multicolumn{2}{|c|}{ Transcription } \\
\hline & $2 \mathrm{~d}$ & & & Ist & $2 d$ \\
\hline $\bar{n}$ & ลิ & $\mathrm{k}$ & $\ldots$ & $\hat{a}$ & o \\
\hline \multirow[t]{2}{*}{2} & $w$ & $\mathrm{kh}$ & $-\therefore$ & $\mathrm{a}^{x}$ & ŭa \\
\hline & w & $\mathrm{ng}$ & $-7-$ & $a$ & ea \\
\hline$\overline{0}$ & $\tilde{\omega}$ & ch & -ר- & $\breve{a}$ & ŏa/ĕab \\
\hline \multirow[t]{2}{*}{$\bar{x}$} & w & $\mathrm{chh} / \mathrm{ch}^{\mathrm{a}}$ & 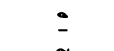 & $\breve{\mathrm{e}}$ & $\breve{\mathbf{i}}$ \\
\hline & $\underset{\sim}{m}$ & $\mathrm{nh}$ & $\simeq$ & ey & $\mathrm{i}$ \\
\hline U & ฌ & $\mathrm{d}$ & $=$ & ŏe & ĕu \\
\hline U, Щू & ก) & th & $=$ & oeu & eu \\
\hline ณ & & $\mathrm{n}$ & i & $\breve{o}$ & $\breve{\mathbf{u}}$ \\
\hline$\hat{n}$ & 6 & $\mathrm{t}$ & $i$ & $\mathrm{au}$ & ou \\
\hline \multirow[t]{2}{*}{$\tilde{u}$} & $\bar{\omega}$ & th & - & uo & uo \\
\hline & s & $\mathrm{n}$ & $\tilde{\xi}$ & aoe & oe \\
\hline$u$ & & $\mathrm{~b}$ & 6-] & eua & eua \\
\hline$\ddot{U}$ & $n$ & $\mathrm{p}$ & $6-9$ & ie & ie \\
\hline \multirow[t]{6}{*}{$\tilde{\omega}$} & $\hat{n}$ & $\mathrm{ph}$ & $6-$ & $\mathrm{e}$ & é \\
\hline & $\forall$ & $\mathrm{m}$ & $i^{2}$ & ae & $\breve{\mathrm{e}}$ \\
\hline & $w$ & $y$ & \} & ai & ei \\
\hline & $J$ & $r$ & $(-)$ & ao & $\breve{o}$ \\
\hline & ญ & 1 & $6-7$ & av & ŏv \\
\hline & $\xi$ & $\mathbf{v}$ & $\doteqdot$ & ŏm & ŭm \\
\hline$\hat{v}$ & & $\mathrm{~s}$ & $\therefore$ & ấm & $\breve{\text { umm }}$ \\
\hline
\end{tabular}


Note on Transliteration

\begin{tabular}{|c|c|c|c|c|}
\hline Consonants & Transcription & Vowels & \multicolumn{2}{|c|}{ Transcription } \\
\hline ist & $2 \mathrm{~d}$ & & Ist & $2 \mathrm{~d}$ \\
\hline ญ & h & $-i$ & ăm & ŏam \\
\hline [) & 1 & $-i$ w & ăng & ĕang \\
\hline H & $\varnothing /{ }^{c}$ & $-:$ & ăh & ĕah \\
\hline
\end{tabular}

aWen followed by another consonant, as in chnăm [year].

before a velar final, as in nĕak [person].

'When subscript to another consonant, as in s'at [clean].

Although this system is similar to that used in many Frenchlanguage works on Cambodia, additional diacritics act to distinguish all of the various vowel sounds. The system is used as printed above, with the following modifications:

I. No unpronounced finals are written.

2. Final $u$ is written $p$. 
Cambodian Culture since 1975 
